\title{
A Study on the Physical Therapy Approach for Improving the Upper Extremity Function of Spastic Ischemic Stroke Patients
}

\author{
Dae-Hwan Lee ${ }^{1}$ and Jung-Ho Lee ${ }^{2}$ \\ ${ }^{1}$ Department of Development, Y. H. Life Co., Ltd., B107, CU techno center, 13-13, \\ Hayang-ro, Hayang-eup, Gyeongsan-si, Gyeongsangbuk-do, Republic of Korea \\ 2 (Corresponding Author) Department of Physical Therapy, Kyungdong Univ., \\ Bongpo-ri, Toseong-myeon, Goseong-gun, Gangwon-do, Republic of Korea \\ 1dhlee8510@naver.com, ${ }^{2}$ ljhcivapt@naver.com
}

\begin{abstract}
This study aimed to understand the conventional physical therapy for improving the upper extremity functions of spastic ischemic stroke. This is a literature study with books, articles, and papers. We consider the upper extremity function of spastic ischemic stroke can be improved with physical therapy which can promote the healing process. To improve the upper extremity functions of the patient, a systematic approach of various methods is required.
\end{abstract}

Keywords: Ischemic stroke, Spasticity, Upper extremity function, Physical therapy

\section{Introduction}

Many people suffer long-term sequelae after stroke and have difficulty performing independent activities because of the loss of functional abilities. Most stroke patients experience impairment of upper extremity motor skills, and $80 \%$ of hemiplegic patients experience hand function disorder [1][2][3]. The deterioration of the upper limb functions in stroke patients is associated with the spasticity of the upper extremities. The spasticity of the upper extremities mostly appears 1 year after stroke, which acts as a critical factor that interferes with the independent life of stroke patients [4]. Spasticity is a general characteristic of upper motor neuron syndrome and ischemic stroke. A fast joint movement in spastic ischemic stroke patients increases the tension of the muscle by stretch reflex, which reinforces the jerk phenomenon of tendons; thus, it is a movement that should be avoided by spastic patients.

According to the Health Insurance Review and Assessment Service, 486,000 patients are receiving treatment for ischemic stroke in South Korea. According to a study by Wizzle, spasticity develops within 6 weeks after onset in one in four stroke patients, primarily in the humeroulnar joint (79\%), radiocarpal joint (66\%), and tibiotarsal joint (66\%) [5]. It has been reported that the spasticity pattern in the upper extremities appeared as medial rotation and adduction of the shoulder, flexion of the elbow, and flexion of the wrist and fingers in the upper extremities, and the abduction and extension of the knee and equinovarus of the foot in the lower extremities [5][6]. Another study reported that spasticity of the upper extremities

Article history:

Received (April 7, 2019), Review Result (May 4, 2019), Accepted (May 29, 2019) 
appears within the first 1 year in $4 \%$ to $38 \%$ of stroke patients, and impairment of the arm function appears in $25 \%$ to $46 \%$ [7][8][9].

Unlike the lower extremities, the upper extremities have many functional aspects and play critical roles in functional daily living activities, such as eating, bathing, dressing, using the toilet, washing the face, and taking a shower, and special functional activities, such as using a computer and writing. Securing the upper extremity function is critical because stroke patients with bilateral paralysis must lead daily life by making the best of the upper extremity functions.

The current treatments applied to stroke patients include medication, Transcutaneous Electrical Nerve Stimulation (TENS), Functional Electrical Stimulation (FES), traditional physical therapy such as stretching to improve the viscoelasticity of muscles, task-oriented training to enhance functional performance, and orthoses to prevent muscle shortening. However, there are still limitations in the physical therapy approach to recovering the upper limb functions of spastic stroke patients. Therefore, this study reexamined various literature because it is necessary to understand the upper extremity functions of spastic stroke patients, and a physical therapy approach is required to recover the upper extremity functions of these patients.

\section{Main discourse}

\subsection{Occurrence of spastic ischemic stroke}

Only a few studies have addressed the occurrence of spasticity. Somerfield [10][11] conducted the Modified Ashworth Scale (MAS) test for 95 first-stroke patients and found that spasticity appeared in $21 \%$ of all patients and $26 \%$ of 77 hemiplegic patients within 5.4 days on average. Furthermore, spasticity appeared within 3 months in $19 \%$ of the 95 subjects and in $28 \%$ of 64 patients who still had hemiplegia. The MAS scores of the patients stayed the same in most cases in 5 days and 3 months after onset, and only one patient changed from 1 to 4 points. These results suggest that the prevalence in all patients did not increase from 5 days to 3 months, but only increased slightly in the hemiplegia group.

According to a recent study, the morbidity rate of spasticity was not much difference between the upper and lower extremities, but severe spasticity cases mostly appeared in the upper extremities [12]. However, severe cases of spasticity are very rare.

\subsection{Pathology of the upper extremities of spastic ischemic stroke patients}

Spasticity in stroke patients is caused by the collapse of the relationship between sensory input and motor response, which leads to the hypersensitive reaction of the compartmentalized central nervous system [12]. This is related to the intensity of the input sensors, such as the strength of stretching, and the location of damage in the central nervous system. If the balance between the inhibitory and excitatory nerve fibers is broken, various syndromes of the upper extremities can appear, such as hypotonia, dyskinesia, and spasticity. Spasticity is caused when there is damage to the brain stem, the primary, secondary, and subsidiary movement regions of the cerebral cortex, and area 3 of the pyramidal tract of the spinal cord [13]. The occurrence of spasticity can be explained by the reorganization of disorderly nerves after the brain is damaged. 


\subsection{Assessment of spastic ischemic stroke patients}

\section{(1) Modified Ashworth Scale}

The MAS is a method of measuring the degree of resistance of passive motions. It cannot measure the passive movement speed of joints, angle of muscle contraction, and degree of potential resistance of tendons. The MAS is an assessment method that can be easily used in clinical practice and is widely used in many studies. However, it showed an average degree of reliability in validation research [14]. The reliability of the MAS is limited if the joint motions are reduced by contracture and are not affected by the joint movement speed. However, the MAS is established for assessing the contracture of the soft tissue and the combination of spastic myopathy and spasm [15][16].

(2) Modified Tardieu Scale

The Modified Tardieu Scale (MTS) is an assessment method for the passive joint motion speed and potential resistance of tendons. This assessment method classifies spasticity into three levels of speed: low, normal, and fast. It is a more accurate indicator than the MAS. The MTS is more sensitive because muscle resistance and speed are measured after muscle contraction [17].

Table 1. Modified Ashworth Scale (MAS)

\begin{tabular}{|c|c|}
\hline Grade & Description \\
\hline 0 & Muscle tone not increased \\
\hline 1 & $\begin{array}{l}\text { The muscle tone is slightly increased and appears as a catch and release or minimum } \\
\text { resistance at the end of the ROM when the affected part is moved to flexion or extension }\end{array}$ \\
\hline $1+$ & $\begin{array}{l}\text { The muscle tone is slightly increased, and appears as a catch, followed by minimal } \\
\text { resistance throughout the remainder (less than half) of the ROM }\end{array}$ \\
\hline 2 & $\begin{array}{l}\text { The increase in muscle tone was noticeable through most ROMs, but the affected part/s } \\
\text { moved easily }\end{array}$ \\
\hline 3 & The muscle tone has increased considerably, making passive movement difficult. \\
\hline 4 & Affected part/s rigidity appears throughout flexion or extension \\
\hline \multicolumn{2}{|r|}{ ROM, range of motion } \\
\hline
\end{tabular}

Table 2. Modified Tardieu Scale (MTS)

\begin{tabular}{|c|c|}
\hline Grade & Description \\
\hline 0 & No resistance throughout the passive movement \\
\hline 1 & Slight resistance throughout the passive movement, without a clear catch at the correct angle \\
\hline 2 & Clear catch at the correct angle, interfering with the passive movement, followed by the \\
release
\end{tabular}




\subsection{Physical therapy of the upper extremity in spastic ischemic stroke patients}

(1) Basics of Physical Therapy

The basic principle of physical therapy for spastic patients is that limiting muscle contracture and reducing over-activity at least for a short period can help them. The purpose of stretching is to enhance the viscoelasticity of muscles and tendons and increase extensibility. However, stretching can increase the tension of the surrounding tissues, such as blood vessels, skin, connective tissues, and nerve tissues. The applied strength, time, speed, and frequency of stretching have not been agreed upon yet. A recent study systematically examined the effect of stretching to remove the muscle contracture of brain-damaged patients and concluded that stretching did not cause a significant change in joint mobility, pain, spasticity, or limitation of activity [18].

Other physical therapy methods are used to reduce spasticity and improve motor function. Hydrotherapy, cryotherapy, hyperthermia, vibration stimulation, inhibition of neurodevelopment, and treatment with a robot are used to relax muscles and lower the intensity of spasticity.

(2) Constraint-Induced Movement Therapy

Constraint-induced movement therapy (CIMT) is an exercise intervention developed based on the learned nonuse model and cortical relocation phenomenon. It enforces the use of the affected side by prohibiting the use of the unaffected side. The unaffected side is restricted for $90 \%$ of the wake time for 2 weeks, and intensive and repeated training is performed on the affected side [18].

The advantage of CIMT is that it can induce improvement of functions and prevent pain and overuse syndrome caused by the overuse of the unaffected side because it allows patients to train themselves, train functional activities, suppress the unaffected side, and force patients to use the affected side. However, CIMT can cause psychological anxiety and burden because it is a high-intensity training program for patients. Furthermore, it is difficult to perform training for 6 hours in treatment facilities. It is also difficult to live everyday life because restriction of the unaffected side and the psychological burden increases as a result.

(3) Orthoses

Orthoses are used to complement and assist physical therapy, reduce spasticity and pain, improve functions, give a sense of protection, and prevent deterioration. Their biggest advantage is that they have a long-duration of effect because patients can be left for several hours without a therapist or nurse. However, orthoses have not been proved through a doubleblind test, and no improvements were observed in the spasticity of the wrist flexion muscle or movement of the entire hand [19].

(4) Transcutaneous Electrical Nerve Stimulation

TENS is a physical therapy method that injects electric signals according to the site of spasticity and the skin nerve control level of the spinal cord. It is effective in reducing the spasticity of the antagonist's muscle. The effect of TENS seems to be associated with the production of $\beta$-endorphin, which reduces the activity of the muscle. According to the gateway control theory, it appears to reduce the input of new information by adjusting the delivery of pain signals. It has been suggested that TENS can promote the reorganization of the cerebral cortex synapses and accelerate the exercise output by increasing the sensory input by stimulating the $A \beta$-tissue of the large-diameter nerve fibers [20]. More research is required 
to define the most effective parameters and describe the mechanism that forms the basis of this theory.

\section{Conclusion}

Geriatric diseases are increasing with the increasing elderly population globally as well as in South Korea. In particular, stroke is a very dangerous disease and is the second most common cause of death among single diseases. Furthermore, stroke causes large individual, social, and economic losses because of serious sequelae that interfere with daily life after survival, and the quality of life of stroke patients is low as a result. The upper extremities are critical not only for the daily life of individuals but also for their social activities, and they are directly related to the workability of individuals. Even though there are many treatments to enhance the upper limb functions of stroke patients, their therapeutic effects are not consistent. In the future, more diverse and systematic approaches to reinforce the arm and hand functions of stroke patients would be necessary.

\section{Acknowledgments}

This work was supported by the National Research Foundation of Korea (NRF) grant funded by the Korean government (MSIT) (No-2019R1F1A1057731).

\section{References}

[1] Lance J.W., "The control of muscle tone, reflexes, and movement: Robert Wartenberg Lecture," Neurology, vol.30, pp.1303-1313

[2] P. Maciejasz, J. Eschweiler, K. Gerlach-Hahn, A. Jansen-Troy, and S. Leonhardt, "A survey on robotic devices for upper limb rehabilitation," Journal of Neuroengineering and Rehabilitation, vol.11, no.1, article no.3, (2014)

[3] C. Yoo and J. Park, "Impact of task-oriented training on hand function and activities of daily living after stroke," Journal of Physical Therapy Science, vol.27, no.8, pp.2529-2531, (2015)

[4] Watkins CL, et al., "Prevalence of spasticity post stroke," Clin Rehabil, vol.16, pp.515-536. article no.7, (2002)

[5] Hefter H, Jost WH, Reissig A, Zakine B, Bakheit AM, and Wissel J., "Classification of posture in post-stroke upper limb spasticity: A potential decision tool for botulinum toxin a treatment?" International Journal of Rehabilitation Research, vol.35, pp.227-233, (2012)

[6] Marciniak C., "Poststroke hypertonicity: Upper limb assessment and treatment," Topics in Stroke Rehabilitation, vol.18, pp.179-194, (2011)

[7] Lundstrom E, Terent A, and Borg J., "Prevalence of disabling spasticity 1 year after first-ever stroke," Eur J Neurol, vol.15, pp.533-539, (2008)

[8] Urban PP, et al., "Occurrence and clinical predictors of spasticity after ischemic stroke," Stroke, vol.41, pp.2016-2020, (2010)

[9] Wissel J, et al., "Early development of spasticity following stroke: a prospective, observational trial," J Neurol, vol.257, pp.1067-1072, (2010)

[10] A. Opheim, et al., "Upper-limb spasticity during the first year after stroke: stroke arm longitudinal study at the University of Gothenburg," Am J Phys Med Rehabil, vol.93, pp.884-896, (2014)

[11] Sommerfeld DK, et al., "Spasticity after stroke: Its occurrence and association with motor impairments and activity limitations," Stroke, vol.35, pp.134-139, (2004)

[12] Mayer NH and Esquenazi A., "Muscle overactivity and movement dysfunction in the upper motoneuron syndrome," Physical Medicine \& Rehabilitation Clinics of North America, vol.14, pp.855-883, (2003) 
[13] Soyuer F and O" ztu"rk A., "The effect of spasticity, sense and walking aids in falls of people after chronic stroke," Disability \& Rehabilitation, vol.29, pp.679-687, (2007)

[14] Ghotbi N, Nakhostin Ansari N, Naghdi S, and Hasson S., "Measurement of lower-limb muscle spasticity: Intrarater reliability of modified ash worth scale," Journal of Rehabilitation Research \& Development, vol.48, pp.83-88, (2011)

[15] Gracies JM, Burke K, Clegg NJ, Browne R, Rushing C, Fehlings D, Matthews D, Tilton A, and Delgado MR., "Reliability of the Tardieu Scale for assessing spasticity in children with cerebral palsy," Archives of Physical Medicine \& Rehabilitation, vol.91, pp.421-428, (2010)

[16] Thakker MM and Rubin PA., "Pharmacology and clinical applications of botulinum toxins A and B," International Ophthalmology Clinics, vol.44, pp.147-163, (2004)

[17] Gracies JM, Marosszeky JE, Renton R, Sandanam J, Gandevia SC, and Burke D., "Short-term effects of dynamic lycra splints on upper limb in hemiplegic patients," Archives of Physical Medicine \& Rehabilitation, vol.81, pp.1547-1555, (2000)

[18] Taub E, Uswatte G., and Pidikiti R., "Constraint-induced movement therapy: A new family of techniques with broad application to physical rehabilitation a clinical review," J Rehabil Res Dev, vol.36, no.3, pp.237251

[19] Basaran A, Emre U, Karadavut KI, Balbaloglu O, and Bulmus N., "Hand splinting for poststroke spasticity: A randomized controlled trial," Topics in Stroke Rehabilitation, vol.19, pp.329-337, (2012)

[20] Yan T and Hui-Chan CW., "Transcutaneous electrical stimulation on acupuncture points improves muscle function in subjects after acute stroke: A randomized controlled trial," Journal of Rehabilitation Medicine, vol.41, pp.312-316, (2009) 